Check for updates

Cite this: Mater. Adv., 2020 1,254

Received 31st March 2020, Accepted 20th April 2020

DOI: 10.1039/d0ma00150c

rsc.li/materials-advances

\title{
Ditch-structured microporous layers fabricated by nanosecond-pulse laser ablation for enhancing water transport in polymer electrolyte membrane fuel cells $\dagger$
}

\author{
Dong-Hyun Lee, ${ }^{a}$ Min Kyung Kim, ${ }^{\mathrm{b}}$ Hwanuk Guim, ${ }^{\mathrm{c}}$ Seongmin Yuk, ${ }^{\mathrm{a}} \mathrm{Jaeho} \mathrm{Choi,}^{\mathrm{a}}$ \\ Sungyu Choi, ${ }^{a}$ Gisu Doo, ${ }^{a}$ Dong Wook Lee, ${ }^{a}$ Jiwhan Noh*d and Hee-Tak Kim (D) *a
}

\begin{abstract}
Water management becomes a more critical issue as the power performance of polymer electrolyte membrane fuel cells (PEMFC) is progressively improved. Herein, we present a ditch-structured microporous layer (MPL) that can prevent water flooding in PEMFCs. In-plane ditch structures are carved on an MPL using a nanosecond-pulse laser ablation technique while preserving the surface porosity of the MPL. When the direction of the ditches is aligned perpendicular to the flow field direction, the power performance is significantly enhanced due to the facilitated mass transport under the rib area. The $i-V$ polarizations and limiting current analysis suggest that not gas transport but water transport is responsible for the power performance enhancement. Compared with a perforated MPL prepared by the same technique, the ditch-structured MPL is more effective in mitigating water flooding. Diagonal and radial ditches exemplify the efficacy in making complex ditch patterns. The delicate structural engineering of the MPL enabled by laser ablation can offer a novel design platform for advanced fuel cells.
\end{abstract}

\section{Introduction}

Fuel cells have been regarded as a promising candidate for zero $\mathrm{CO}_{2}$ emission power generation systems. Among the various fuel cell types, polymer electrolyte membrane fuel cells (PEMFCs) are closer to mass-commercialization due to their high power density and moderate operation temperature. ${ }^{1-3}$ During the last few decades, PEMFC research has shown significant progress in improving the power performance, including advanced catalyst and membrane materials, electrode structures, and flow field designs. ${ }^{4-7}$ These advancements enable PEMFCs to operate at higher current densities; however, they impose a substantial burden on removing water, the amount of which is proportional to the current density. The accumulation of product water often causes water flooding, resulting in a large polarization or a

\footnotetext{
${ }^{a}$ Department of Chemical \& Biomolecular Engineering, Korea Advanced Institute of Science and Technology (KAIST), 291, Daehak-ro, Yuseong-gu, Daejeon, 34141, Republic of Korea. E-mail: heetak.kim@kaist.ac.kr

${ }^{b}$ Department of Materials Science and Engineering, Korea University, Seoul, 02841, Republic of Korea

${ }^{c}$ Division of Electron Microscopic Research, Korea Basic Science Institute, Daejeon, 34133, Republic of Korea

${ }^{d}$ Korea Institute of Machinery \& Materials (KIMM), Jang-dong 171, Yuseong-gu, Daejeon,34101, Korea.E-mail: njw733@kimm.re.kr

† Electronic supplementary information (ESI) available. See DOI: 10.1039/ d0ma00150c
}

sudden drop in operating voltage..$^{8-11}$ Therefore, for further progress of high-power PEMFCs, advanced water management technologies are required.

Regarding water management, the microporous layer (MPL) has a critical role. It consists of carbon and a hydrophobic binder such as PTFE and is placed in between the catalyst layer (CL) and gas diffusion layer (GDL). ${ }^{12-17}$ Due to its mesoporous structure and hydrophobic binder, the MPL facilitates water removal from the CL to the carbon paper and provides unsaturated pores for gas transport even in the event of liquid water condensation. In a PEMFC stack, the bipolar plates with a flow channel and rib compress the GDL and MPL by the stack pressure applied to it to ensure electrical contact and a tight seal. As a result, under the rib area, most of the MPL pores are saturated with water, forming a sharp boundary of water saturation at the interface between the ribs and channels, ${ }^{18,19}$ which has been regarded as an inherent problem of the current PEMFC structures. Structural modifications of the GDL and $\mathrm{CL}$ have been attempted to remove the water under the rib area. ${ }^{20-23}$ Naing et al. suggested a GDL with an anisotropic fiber arrangement,$^{22}$ and our group reported on a patterned CL with in-plane micron channels in it. ${ }^{23}$ Both approaches enhance the power performance in the high current density regime when the direction of the in-plane mass transport is perpendicular to the flow channel direction, emphasizing the importance of the in-plane guiding of water removal. 
(a)

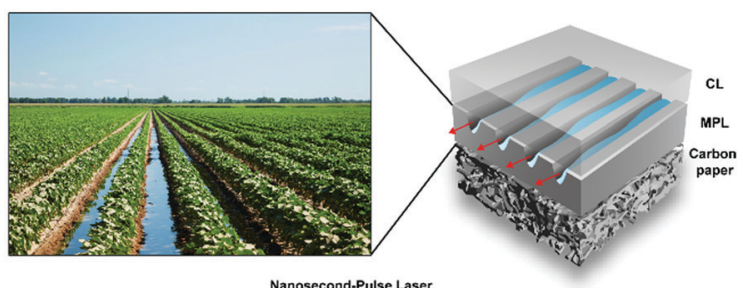

(b) Nanosecond.Pulse Laser

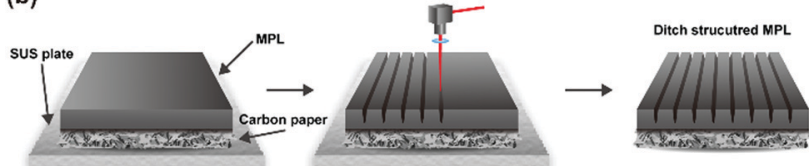

Fig. 1 Schematics of (a) in-plane water transport through the ditchstructure carved on the MPL in analogy with water drainage through field ditches (inset image) and (b) fabrication process for making ditches on the MPL surfaces by scanning a nanosecond-pulse laser.

Prevention of water flooding is also one of the crucial issues in the architecture. The simplest solution developed so far in the architecture to prevent water flooding was to make a ditch. Inspired by the guided water flow by the ditch, herein we present a ditch-structured MPL to remove the water under the rib area effectively. Fig. 1a illustrates the overall concept and structure developed in this work. Making ditch structures in the MPL has not been attempted due to the difficulty of their fabrication. However, we achieved manufacturing a well-defined ditch structure carved on the MPL using a nanosecond-pulse laser ablation technique, which is widely using for the patterning of metal surfaces. ${ }^{24-28}$ Previously, in the PEFMC field, the laser perforation technique has been used to form through-plane holes of approximately $100 \mu \mathrm{m}$ diameter inside the GDL for water removal. ${ }^{29-33}$ Our ditch structure is different from the perforated structure in that the ditches guide not through-plane but in-plane mass transport. Furthermore, various ditch structures were simply fabricated, like drawing a line with a laser. The nanosecond-pulse was selected considering the polymer binder melting problem with the high power laser perforation technique reported in previous work. ${ }^{29}$ The nanosecond-pulse laser ablation used in this work can decompose the materials under a short-pulse duration, which is beneficial in preserving the surface pores on the ditch structure. Additionally, the nanosecond-pulse laser is more cost-effective and suitable for commercialization compared to well-known short-pulse lasers, the pulse duration of which is in the pico or femtosecond range. We demonstrate that the in-plane-ditched MPL structure can effectively mitigate water flooding under the rib area and is even more advantageous than the perforated structure.

\section{Experimental}

\section{Surface laser irradiation}

The ditch structure was fabricated using a high-power Q-switched diode-pumped UV laser (JDSU Q302-HD) system and a scanning system. An MPL-coated GDL (SIGRACET 39BC, SGL carbon SE) was used for the ditch formation. The focal length between the laser source and the MPL surface was $6.5 \mathrm{~mm}$; the power output was $0.7 \mathrm{~W}$, and the repetition rate was $20 \mathrm{kHz}$.

\section{Ditch structure characterization}

After the ablation, the ditch structures were observed with field emission-scanning electron microscopy (FE-SEM, Sirion, FEI). The chemical structures of the MPL surface before and after laser ablation were characterized by using X-ray photoelectron spectroscopy (Sigma Probe, Thermo VG Scientific). Optical images of the water droplet were obtained with a laser scanning confocal microscope (LSM 5 PASCAL, Carl Zeiss). The contact angles of the water droplet were measured using a goniometer (DSA 10-Mk2, KRUSS), in which $5 \mu \mathrm{L}$ drops were deposited.

\section{Single-cell fabrication and operation}

A commercial carbon-supported Pt catalyst (TEC10F50E, Tanaka Kikinzoku Kogyo), a Nafion ionomer dispersion (D520, Dupont) and a water/dipropylene glycol binary solvent (1/1 by volume) were homogeneously mixed by ball-milling for $3 \mathrm{~h}$. The resulting catalyst slurry was cast onto an FEP film and dried at $60{ }^{\circ} \mathrm{C}$ for $12 \mathrm{~h}$ to form the anode and cathode CL. To fabricate the catalyst coated membrane (CCM), a stack consisting of the anode CL, a $25 \mu \mathrm{m}$-thick membrane (NRE211, Dupont), and the cathode CL was pressed at $20 \mathrm{~atm}$ and $130{ }^{\circ} \mathrm{C}$ for $5 \mathrm{~min}$. The FEP films were peeled off from the laminate. The cathode and anode CL had Pt loadings that were

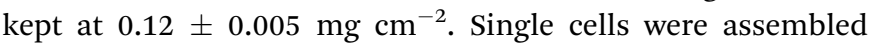
using the CCMs with an active area of $25 \mathrm{~cm}^{2}, 39 \mathrm{BC}$ as an anode GDL and 39 BC or ditched 39 BC as a cathode GDL, and graphite plates with a single-serpentine flow field. The air and hydrogen feeds were supplied in a co-flow configuration.

\section{Electrochemical analysis}

$I V$ polarization tests were conducted with a fuel cell test station (Scitech Korea) at $65{ }^{\circ} \mathrm{C}$ with a stoichiometric ratio of $\mathrm{H}_{2}$ /air or $\mathrm{O}_{2}$ of 1.2/1.5 and various relative humidity conditions without backpressure. Electrochemical impedance spectroscopy (EIS) and cyclic voltammetry measurements were done with a potentiostat (HCP-803, BioLogics Science Instruments).

\section{Results and discussion}

In the fabrication of the ditch structures on the MPL, the control of the ablation conditions is essential for the precise structure. As shown in the schematic of the fabrication process (Fig. 1b), the laser beam irradiates while moving along a programmed pathway at a constant speed. The power of the laser was carefully adjusted to have a high aspect ratio for the ditches. The distance between the sample and the scan head was finely tuned to locate the focal point of the laser beam on the MPL surface.

The number of laser scans can vary the depth and width of the ditch. The ditch structure becomes deeper and broader by increasing the scan number. Typical single and triple scan ablation were compared. Fig. 2a-c show the cross-sectional and 

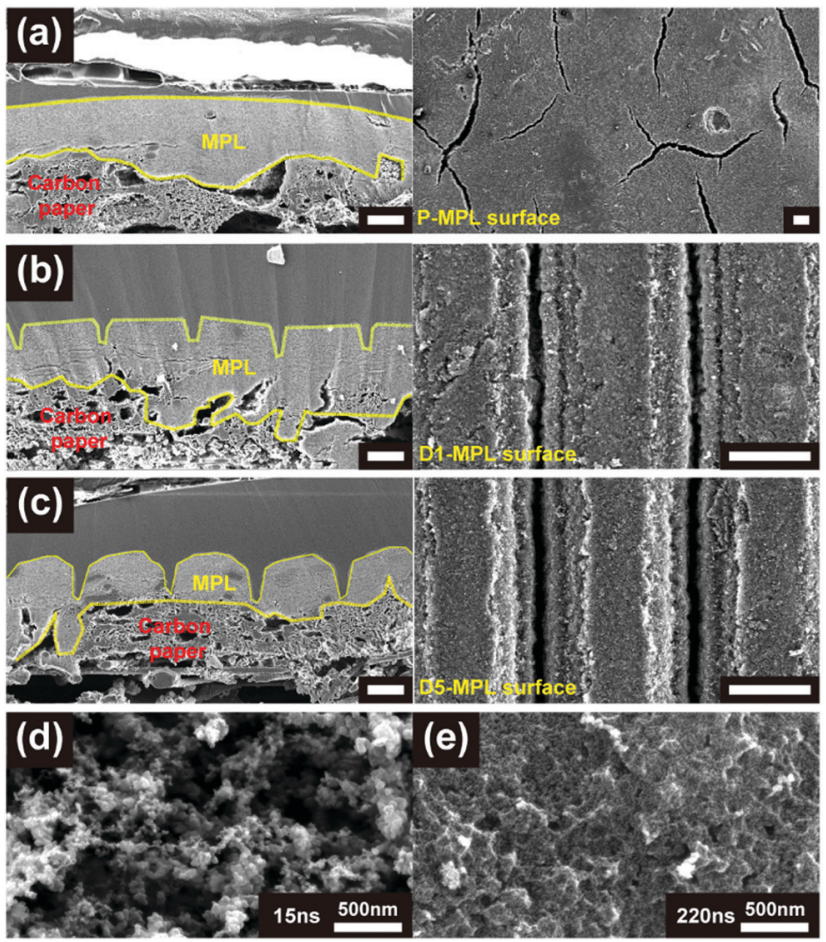

Fig. 2 SEM images of the MPL after laser ablation. (a) Cross-sectional view (left) and MPL-side view (right) of the pristine GDL consisting of the MPL (P-MPL) and carbon paper. Cross-sectional view (left) and MPL-side view (right) of the GDLs after (b) the single laser scan (D1-MPL) and (c) triple laser scan (D3-MPL). Yellow lines indicate the boundary of the MPLs. (Inset scale bar: $50 \mu \mathrm{m})$. SEM images of the side wall of the ditch prepared with a pulse duration of (d) $15 \mathrm{~ns}$ and of (e) 220 ns.

top-view SEM images for the pristine MPL (P-MPL), MPL with single ablation (D1-MPL), and MPL with triple ablation (D3-MPL), respectively. The specimens for the cross-sectional SEM measurement were prepared with epoxy filler to prevent any structural variation during the ion milling process. The cross-sectional SEM images show that the depth of the ditch was approximately 30 and $60 \mu \mathrm{m}$ for the single and triple ablation, respectively. As compared in Fig. $2 \mathrm{~b}$ and c, the opening of the ditch was widened with the repeated ablation. From the SEM images of the top MPL surfaces, the widened opening of the ditches with the repeated ablation was verified again. Moreover, a regular spacing of $100 \mu \mathrm{m}$ was identified for both the single and triple ablation.
A critical issue in the laser ablation of the MPL is the possibility of blocking the surface pores due to the melting of the polymer binders during the laser ablation process. Therefore, it is vital to check the pore structure at the sidewall of the ditch. For a pulse duration of 15 ns, as shown in Fig. 2d, the pore structures are well preserved on the surface of the ditch walls, indicating that the wall surface of the ditch structure does not block the gas or water transport. However, for a pulse duration of $220 \mathrm{~ns}$, the pore structures on the surfaces of the ditch walls are blocked by melted binder (Fig. 2e). The preservation of the porous structure is attributed to the use of the short-pulse laser, which decomposes the polytetrafluoroethylene (PTFE) binder material rather than melting it. This phenomenon indicates that the nanosecond pulse induces photochemical ablation rather than photothermal ablation. The two ablation modes are different in their chemical bond breakage mechanism. For the photothermal model, the energy of the absorbed laser is converted to thermal energy, causing thermal bond breakage. However, in the photochemical model, the bonds are broken in dissociative states because the absorbed photons directly excite the chromophores. ${ }^{34}$ The short-pulse laser prevents the conversion of the absorbed energy into the vibration mode, thus inducing an explosion instead of heating the solid locally. ${ }^{35}$

Because oxygen radicals form during the laser ablation, the chemical structure of the ditch surface can be varied. Fig. 3a shows the XPS spectra for the ditch surface; the carbonyl group $(\mathrm{C}=\mathrm{O})$ and $\mathrm{C}-\mathrm{O}$ signals were notably increased with the laser ablation, indicating that the ditch surfaces are more hydrophilic than the pristine surface. The water contact angles of the P-MPL surface were measured to be $130^{\circ}$ in both the $x$ and $y$ directions, and a water droplet had a symmetrical spherical shape in its optical microscope image (Fig. 3b). However, a water droplet on the D1-MPL surface was ellipsoidal, and the contact angles were different in the measured directions as $110^{\circ}$ (perpendicular to the ditch direction) and $120^{\circ}$ (parallel to the ditch direction) (Fig. 3c). Although the contact angle of D1-MPL varies depending on the measured direction, it was smaller than the pristine MPL, confirming the hydrophilic property of the ditches.

The anisotropic feature of D1-MPL provides preferential water flow along the ditch direction. A water droplet was dripped onto the MPL at $60^{\circ}$ tilted from the ground to identify the guided water transport along the ditch. For D1-MPL, the
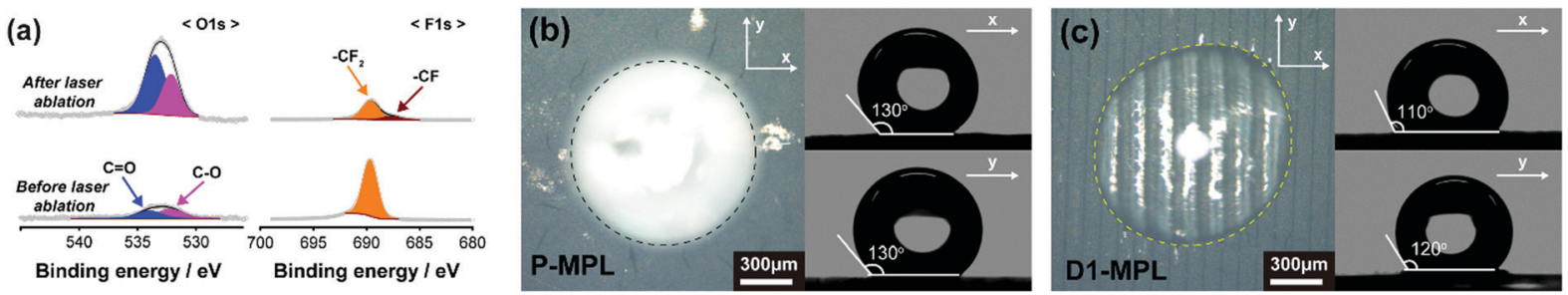

Fig. 3 Surface characteristic of D-MPL. (a) XPS O1s and F1s spectra of the MPL surfaces before and after MPL laser ablation (single scan). Top-view optical image and contact angles in different directions of the water droplet on (b) P-MPL and (c) D1-MPL. The direction of the measured contact angles is indicated by the white mark. 
parallel and perpendicular orientation of the ditches to the ground are denoted as D1-MPL_PA and D1-MPL_PE, respectively. Movie S1 (front-view) and S2 (side-view) (ESI $\dagger$ ) show how the water droplet moved on P-MPL, D1-MPL_PA, and D1-MPL_PE. Fig. S1a-c (ESI $\dagger$ ) show the captured images after disposing of the water droplet for P-MPL, D1-MPL_PA, and D1-MPL_PE, respectively. For P-MPL, the water droplet quickly slid off the surface because of its hydrophobic nature (Fig. S1a, ESI $\dagger$ ). When the direction of the gravity-driven flow and the ditch direction are perpendicular (D1-MPL_PA) the water droplet remained hanging on the surface due to the pinning of the drop in the ditches (Fig. S1b, ESI $\dagger$ ). The ditch structure became visible under the water drop because of the refractive index contrast between the water-filled ditches and the un-ditched surfaces. On the other hand, the ditches oriented in the gravity direction (D1_MPL_PE) enabled the water drop to move down, leaving behind trapped water in the ditches (Fig. S1c, ESI $\dagger$ ). These results indicate that the ditches readily drag water from the droplet due to their hydrophilic property.

The in-plane mass transport across the rib area during the cell operation was investigated by comparing the power performances for the parallel and perpendicular orientations of the in-plane channel to the channel direction in the bipolar plate. For the parallel alignment (D1-MPL_PA), the ditches do not connect the channel and rib areas. Thus, they may not help with water removal from the rib area. By contrast, for the perpendicular alignment (D1-MPL_PE), water can move from the rib area to the channel area along the ditches connecting the two regions.

Before analyzing the effect of the ditch structures on the mass transport, we needed to confirm that the other properties except for the mass transport property can be excluded in the analysis and thus compared the ohmic resistance $\left(R_{\mathrm{Ohm}}\right)$, proton conduction resistance in the CL $\left(R_{\mathrm{CL}}\right)$, and electrochemical surface area (ECSA) for the P-MPL, D1-MPL_PE, and D1-MPL_PA cells. The AC impedances measured in the opencircuit condition (OCV), which represent the values for $R_{\mathrm{Ohm}}$ and $R_{\mathrm{CL}}$, are nearly identical among the three cells (Fig. S2a, $\mathrm{ESI} \dagger)$. Because $R_{\mathrm{Ohm}}$ includes the contribution from electron conduction through the MEA, the invariant $R_{\mathrm{Ohm}}$ implies that the laser-ablated MPL provides sufficient electrical pathways despite the voids on the surface. The electrochemical surface areas (ECSA) for the MEAs were also identical as shown in the $\mathrm{CV}$ responses (Fig. S2b, ESI $\dagger$ ), indicating that these cells have the same catalytic activities.

Fig. 4a shows the $i-V$ polarization curves for P-MPL, D1-MPL_PE, and D1-MPL_PA, measured at a RH of $100 \%$ and ambient pressure with a stoichiometry of 1.2/1.5 for $\mathrm{H}_{2}$ /air. Below $0.6 \mathrm{~A} \mathrm{~cm}^{-2}$, all three cells showed similar power performances, which indicates that their kinetic and ohmic polarizations were nearly identical as expected from the identical $R_{\mathrm{Ohm}}$, $R_{\mathrm{CL}}$, and ECSA values. Above $0.6 \mathrm{~A} \mathrm{~cm}^{-2}$, the power performance increased in the following order: D1-MPL_PA $<$ P-MPL $<$ D1-MPL_PE, which means a decreased mass transport (a)

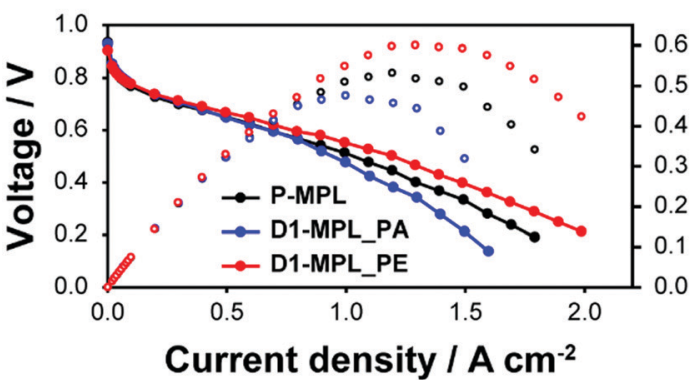

(c)

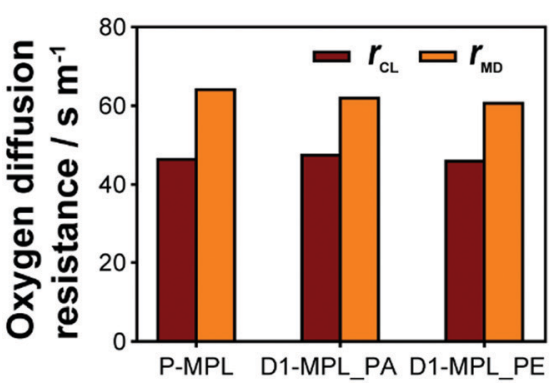

(b)

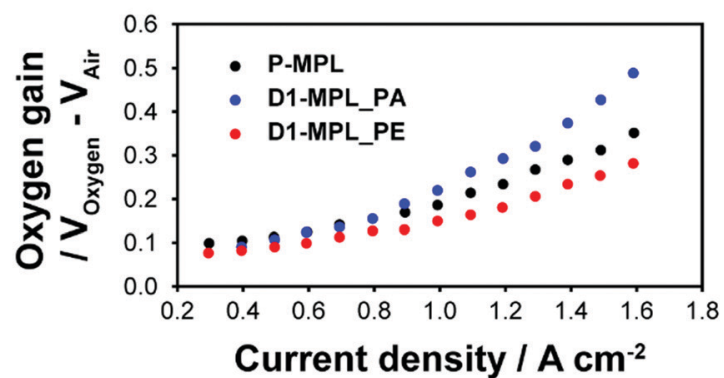

(d)

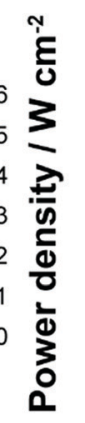

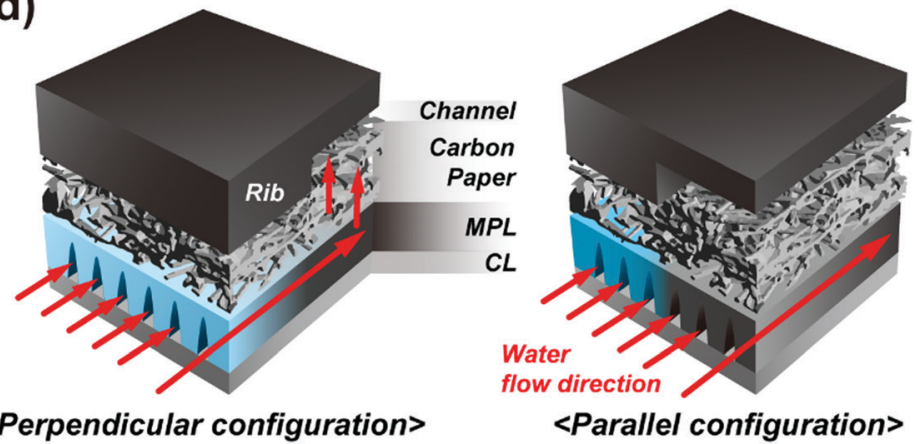

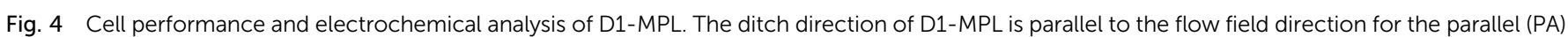

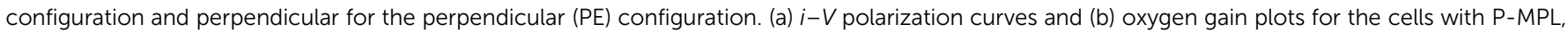

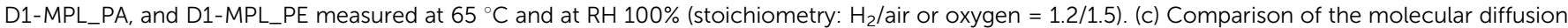

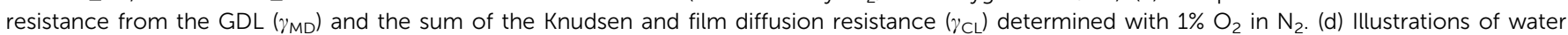
transport for the perpendicular and parallel cell configurations. 
polarization in the same order. It was further confirmed by comparing the oxygen gains (cell voltage differences between $\mathrm{O}_{2}$ and air feed) (Fig. 4b). For the three cells, the oxygen gains exponentially increased with the current density due to an increase in the mass transport polarization. In line with the $i-V$ polarization data, the oxygen gains decreased in the order of D1-MPL_PA > P-MPL > D1-MPL_PE. These results demonstrate that the ditch structure aggravates the mass transport for the parallel arrangement, whereas it facilitates the mass transport for the perpendicular arrangement. The larger transport polarizations with the parallel ditches mean that the hydrophilic voids cause severer water stagnation under the rib area at high RH operation, which was frequently observed for hydrophilic MPLs. ${ }^{36,37}$ However, the significant drop of transport polarization with the switch from the parallel to perpendicular alignment clearly shows that the ditch structure facilitates mass transport between the rib and channel areas.

The reduced transport polarization can be attributed to either gas transport or water transport or both. One of the ways to identify the gas transport property and exclude the water flooding effect is the limiting current method, which features the use of highly diluted oxygen feeds $\left(1 \% \mathrm{O}_{2}\right.$ in $\mathrm{N}_{2}$ and $1 \% \mathrm{O}_{2}$ in $\left.\mathrm{He}\right)$ for quantifying various gas transport resistances; the current densities obtained with the diluted gases are low enough to exclude the effect of water flooding. ${ }^{38,39}$ The total gas transport resistance $\left(\gamma_{\text {Total }}\right)$ determined from the limiting current includes two contributions: the molecular diffusion resistance $\left(\gamma_{\mathrm{MD}}\right)$ from the GDL and the sum of the Knudsen and film diffusion resistances $\left(\gamma_{\mathrm{CL}}\right)$ from the CL. $\gamma_{\mathrm{MD}}$ and $\gamma_{\mathrm{CL}}$ are quantified by measuring the limiting current with the two diluted $\mathrm{O}_{2}$ balance gases. The values for $\gamma_{\mathrm{MD}}$ and $\gamma_{\mathrm{CL}}$ for the three cells are compared in Fig. 4c. The values for $\gamma_{\mathrm{CL}}$ were nearly identical for the three cells, which can be explained by the use of the same CL. Interestingly, the values for $\gamma_{\text {MD }}$ were also similar among the cells despite the structural difference in the MPL. Therefore, gas transport resistance through the ditches is not a significant attribute of the mass transport polarization. The $i-V$ polarization curves of P-MPL and D1-MPL_PE in a low relative humidity condition of $30 \%$ (Fig. S3, ESI $\dagger$ ) also support the indifference of the gas transport; these cells show similar polarization curves because water flooding is insignificant and does not affect the performance at low RH.

According to the limiting current analysis and $i-V$ polarization curves in the low humidity condition, it can reasonably be concluded that the enhanced mass transport with the ditchstructured MPL is attributed to the enhanced water transfer from the rib to the channel area. As shown in Fig. 4d, the hydrophilic ditches, which connect the rib and channel areas, can act as drainage ditches for liquid water, mitigating the water flooding in the rib area. However, in the parallel orientation, such in-plane water transport is not possible because the ditches do not cross the rib and channel area. Instead, the hydrophilic ditches can be a preferred site for water condensation, and the stagnant water in the ditches blocks the gas transport to the nearby catalyst layers. This result provides an important practical implication that not only the structure but also the orientation of the structure must be carefully designed. (a)

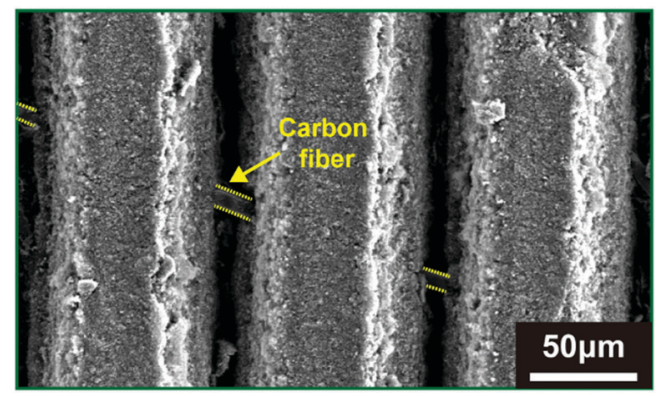

(b)

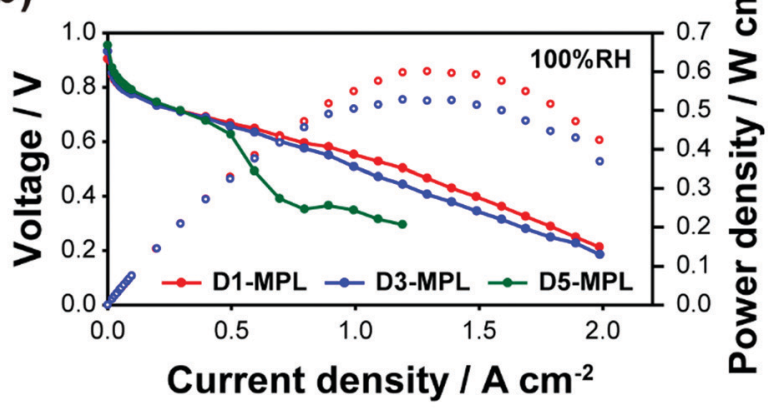

(c)

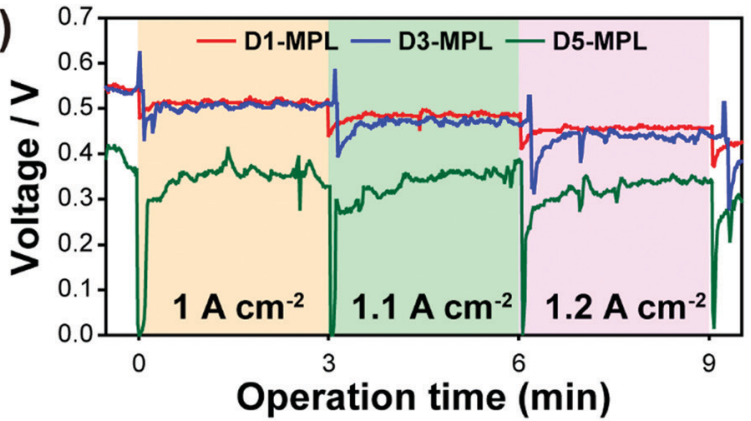

(d)

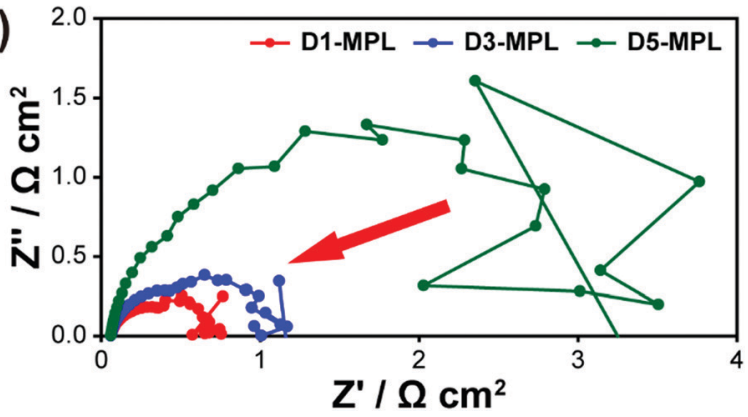

Fig. 5 Cell performance and electrochemical analysis of various D-MPLs with different numbers of laser scans (D1-, D3-, and D5-MPL) at $65^{\circ} \mathrm{C}$ and $\mathrm{RH} 100 \%$. (a) Top-view SEM image of the perforated MPL (D5-MPL). (b) $i-V$ polarization curves, (c) voltage profiles during the high current density operation, and (d) Nyquist plots of the impedances at $1 \mathrm{~A} \mathrm{~cm}^{-2}$ for the cells with D1-, D3-, and D5-MPL. For all the cells, the ditch direction was perpendicular to the flow field direction.

The next question regarding the structural engineering of the MPL is the effect of the ditch depth on the power performance. We compared the ditched MPLs with various ditch depths by varying the laser scan number. With five laser scans, a perforated structure was formed, which is denoted as D5-MPL. For D5-MPL, the underlying carbon fibers of the GDL appear in the top view of the SEM image (Fig. 5a), 
which confirms the perforation. Fig. 5b compares the $i-V$ polarization curves for the D1-, D3-, and D5-MPL cells at a $\mathrm{RH}$ of $100 \%$. The orientations of all the samples were perpendicular to the channel direction. At a $\mathrm{RH}$ of $100 \%$, the power performance of D1-MPL was higher than that of D3-MPL. However, D5-MPL, which is the perforated structure, showed a sudden voltage drop originating from severe water flooding that occurs at the massive void of the ditch structure. In terms of preventing water flooding, it is clear that the ditch structure is more effective than the perforated structure. This result can be explained in terms of the water transport through the MPL in the form of liquid or vapor. ${ }^{40,41}$ The hydrophobic MPL induces liquid water breakage into smaller droplets at the CL/MPL interface, facilitating water evaporation. Due to the lower water saturation level in the MPL, water vapor can be readily transported through the MPL. In addition, the liquid water pressure is augmented at the CL/MPL interface due to the hydrophobicity of the MPL, which is the driving force for capillary transport of liquid water through the MPL or water back flow. ${ }^{15}$ Perforated pores are known to be a preferred liquid water transport channel; ${ }^{42}$ however, the hydrophilic perforated sites in D5-MPL do not have the above-mentioned positive functions of a hydrophobic MPL, in contrast to D1- and D3-MPL, resulting in the inferior water management.

Further experimental results support that the ablated MPL exhibits better water management compared to the perforated MPL. The voltage fluctuation can be used to monitor the water flooding behaviour during a constant current operation, which reflects water accumulation and its prompt removal. Fig. 5c shows the voltage-time profiles along the three steps during the performance measurement for all cases. The D3- and D5-MPL voltage profiles were unstable during the measurement. By contrast, the D1-MPL cell showed a stable profile regardless of the current density increments, which demonstrates effective water management. Another piece of evidence for better water management is the electrochemical impedance spectroscopy (EIS) results at $1 \mathrm{~A} \mathrm{~cm}^{-2}$ shown in Fig. $5 \mathrm{~d}$. The diameter of the low-frequency semi-circle corresponds to the mass transport resistance, and D1-MPL has a smaller semi-circle than that of D5-MPL. The noise and unstable form of the D5-MPL semi-circle indicate accumulated water removal, which demonstrates water flooding. Therefore, the ablated MPL improves the water management compared to the perforated MPL evident by the distinct and smaller low-frequency semicircle of D1-MPL.

The merit of the laser ablation technique is to build a complex ditch structure in a simple manner, which enables systematic and delicate variation of ditch structures. To exemplify the flexibility in the ditch design, we fabricated DI-MPLs with various ditch spacings and complex diagonal and radial ditches. Fig. S4a (ESI $\dagger$ ) shows the performances of D1-MPL with different spacings. The $100 \mu \mathrm{m}$ spacing had a higher power performance than the 30 and $500 \mu \mathrm{m}$ spacing because of a balance between increased hydrophilicity and an increased water removal capability with the reduced ditch spacing. However, the optimum spacing will vary depending on the intrinsic mass transport characteristic of the pristine MEA and the operating conditions. Diagonal and radial ditches were intended to drag water from a relatively-flooded region near the cathode outlet to a relatively dry part near the inlet. These patterns were successfully carved on the MPL, as shown in Fig. 6a,
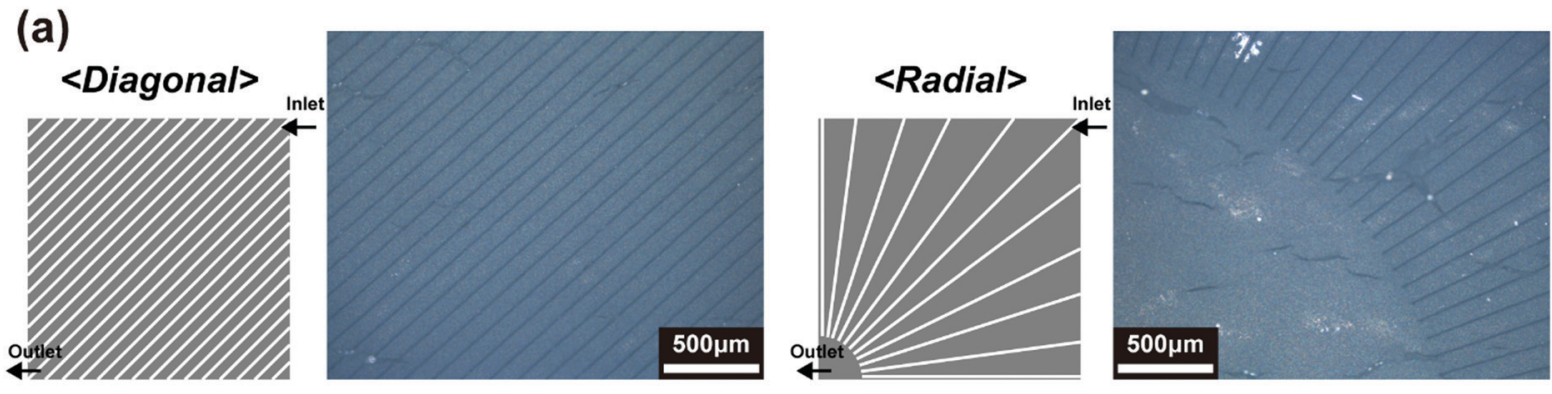

(b)

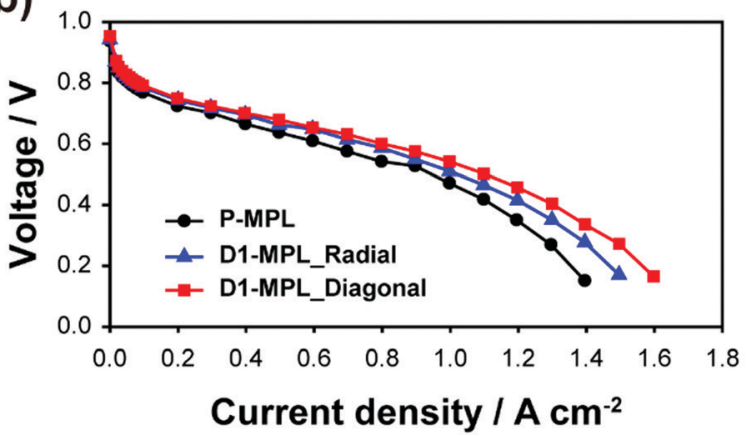

(c)

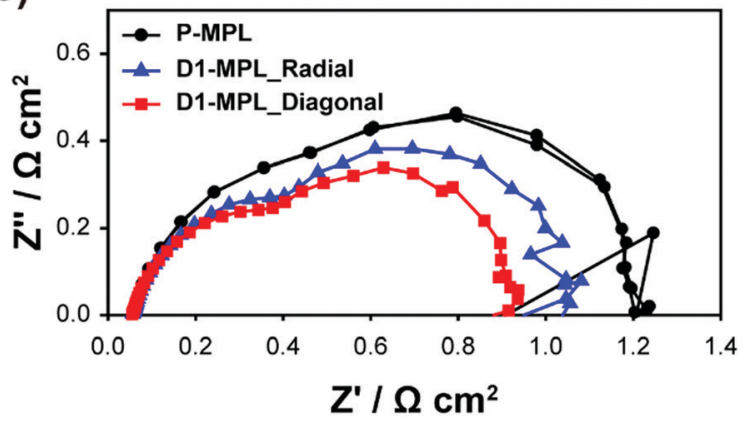

Fig. 6 Cell performance and electrochemical analysis of diagonal and radial ditch structures fabricated with a single laser scan (D1-MPL_Radial and D1-PL_Diagonal, respectively). (a) Schematic illustrations and optical microscope images of the diagonal and radial ditch-structured MPLs. (b) $i-V$ polarization curves at $65^{\circ} \mathrm{C}$ and $\mathrm{RH} 100 \%$ and (c) Nyquist plots of the impedance measured at $1 \mathrm{~A} \mathrm{~cm}^{-2}$ for P-MPL, D1-MPL_Radial, and D1-PL_Diagonal. 
demonstrating the efficacy in making intricate ditch patterns. As shown in Fig. 6b, both diagonal and radial structures perform better compared to P-MPL. It indicates that the ditches connecting more and less flooded regions help to relieve water flooding. EIS analysis also supports the positive effect in reducing the mass transport resistance; the low-frequency semicircles of the diagonal and radial ditches were smaller than that of P-MPL (Fig. 6c). Even though the diagonal ditch showed a better power performance, in this case it does not mean that the diagonal structure is superior. A more sophisticated structural design can promise further improvement for every ditch structure. The examples emphasize that it is possible to create more advanced ditch structures by exploiting nanosecond-pulse laser ablation.

\section{Conclusions}

In summary, using the nanosecond-pulse laser ablation technique, ditch structures were successfully carved on the MPL surface, without losing the surface porosity. When the ditches were aligned perpendicular to the channel direction, the power performance showed notable improvement. The $i V$ polarizations at different RHs and the limiting current density analysis showed that the performance gain is attributed to improved water management. Compared with the perforated MPL, the ditched MPL was more effective in mitigating water flooding and improving the power performance. This work suggests that delicate structural engineering of the MPL can further advance high-power PEMFCs.

\section{Conflicts of interest}

There are no conflicts to declare.

\section{Acknowledgements}

This research was supported by the Technology Development Program to Solve Climate Changes of the National Research Foundation (NRF) funded by the Ministry of Science, ICT \& Future Planning (NRF-2015M1A2A2056733) and the Korea Institute of Energy Technology Evaluation and Planning (KETEP) and the Ministry of Trade, Industry \& Energy (MOTIE) of the Republic of Korea (No. 20173010032100) and a grant from the Korean government-funded Research Project NK217C (the main research project of the Korea Institute of Machinery and Materials).

\section{Notes and references}

1 E. Ticianelli, C. Derouin, A. Redondo and S. Srinivasan, J. Electrochem. Soc., 1988, 135, 2209-2214.

2 B. C. Steele and A. Heinzel, Materials For Sustainable Energy: A Collection of Peer-Reviewed Research and Review Articles from Nature Publishing Group, World Scientific, 2011, pp. 224-231.
3 O. Z. Sharaf and M. F. Orhan, Renewable Sustainable Energy Rev., 2014, 32, 810-853.

4 S. Shamim, K. Sudhakar, B. Choudhary and J. Anwar, Adv. Appl. Sci. Res., 2015, 6, 89-100.

5 B. Lim, E. Majlan, W. R. W. Daud, T. Husaini and M. I. Rosli, Ionics, 2016, 22, 301-316.

6 J. Stacy, Y. N. Regmi, B. Leonard and M. Fan, Renewable Sustainable Energy Rev., 2017, 69, 401-414.

7 E. Majlan, D. Rohendi, W. R. W. Daud, T. Husaini and M. Haque, Renewable Sustainable Energy Rev., 2018, 89, 117-134.

8 S. Shimpalee, U. Beuscher and J. Van Zee, Electrochim. Acta, 2007, 52, 6748-6754.

9 H. Li, Y. Tang, Z. Wang, Z. Shi, S. Wu, D. Song, J. Zhang, K. Fatih, J. Zhang and H. Wang, J. Power Sources, 2008, 178, 103-117.

10 P. Pei, Y. Li, H. Xu and Z. Wu, Appl. Energy, 2016, 173, 366-385. 11 S. Choi, S. Yuk, D.-H. Lee, G. Doo, D. W. Lee, M.-J. Choo and H.-T. Kim, Electrochim. Acta, 2018, 268, 469-475.

12 E. Passalacqua, F. Lufrano, G. Squadrito, A. Patti and L. Giorgi, Electrochim. Acta, 1998, 43, 3665-3673.

13 A. Z. Weber and J. Newman, J. Electrochem. Soc., 2005, 152, A677-A688.

14 S. Park, J.-W. Lee and B. N. Popov, J. Power Sources, 2008, 177, 457-463.

15 Y. Tabe, Y. Aoyama, K. Kadowaki, K. Suzuki and T. Chikahisa, J. Power Sources, 2015, 287, 422-430.

16 J. Zhou, S. Shukla, A. Putz and M. Secanell, Electrochim. Acta, 2018, 268, 366-382.

17 P. Deevanhxay, T. Sasabe, S. Tsushima and S. Hirai, Electrochem. Commun., 2013, 34, 239-241.

18 J. Lee, R. Yip, P. Antonacci, N. Ge, T. Kotaka, Y. Tabuchi and A. Bazylak, J. Electrochem. Soc., 2015, 162, F669-F676.

19 S. S. Alrwashdeh, I. Manke, H. Markötter, M. Klages, M. Göbel, J. Haußmann, J. Scholta and J. Banhart, ACS Nano, 2017, 11, 5944-5949.

20 H. Markötter, K. Dittmann, J. Haußmann, R. Alink, D. Gerteisen, H. Riesemeier, J. Scholta, J. Banhart and I. Manke, Electrochem. Commun., 2015, 51, 133-136.

21 I. Bae, B. Kim, D.-Y. Kim, H. Kim and K.-H. Oh, Renewable Energy, 2020, 146, 960-967.

22 K. S. S. Naing, Y. Tabe and T. Chikahisa, J. Power Sources, 2011, 196, 2584-2594.

23 D.-H. Lee, W. Jo, S. Yuk, J. Choi, S. Choi, G. Doo, D. W. Lee and H.-T. Kim, ACS Appl. Mater. Interfaces, 2018, 10, 4682-4688.

24 B. N. Chichkov, C. Momma, S. Nolte, F. Von Alvensleben and A. Tünnermann, Appl. Phys. A: Mater. Sci. Process., 1996, 63, 109-115.

25 M. Knowles, G. Rutterford, D. Karnakis and A. Ferguson, Int. J. Adv. Des. Manuf. Technol., 2007, 33, 95-102.

26 F. Chen, D. Zhang, Q. Yang, J. Yong, G. Du, J. Si, F. Yun and X. Hou, ACS Appl. Mater. Interfaces, 2013, 5, 6777-6792.

27 D. V. Ta, A. Dunn, T. J. Wasley, R. W. Kay, J. Stringer, P. J. Smith, C. Connaughton and J. D. Shephard, Appl. Surf. Sci., 2015, 357, 248-254. 
28 A. He, W. Liu, W. Xue, H. Yang and Y. Cao, Appl. Surf. Sci., 2018, 434, 120-125.

29 D. Gerteisen, T. Heilmann and C. Ziegler, J. Power Sources, 2008, 177, 348-354.

30 D. Gerteisen and C. Sadeler, J. Power Sources, 2010, 195, 5252-5257.

31 H. Markötter, R. Alink, J. Haußmann, K. Dittmann, T. Arlt, F. Wieder, C. Tötzke, M. Klages, C. Reiter and H. Riesemeier, Int. J. Hydrogen Energy, 2012, 37, 7757-7761.

32 Z. Lu, J. Waldecker, M. Tam and M. Cimenti, ECS Trans., 2015, 69, 1341-1353.

33 X. Wang, S. Chen, Z. Fan, W. Li, S. Wang, X. Li, Y. Zhao, T. Zhu and X. Xie, Int. J. Hydrogen Energy, 2017, 42, 29995-30003.

34 M. Sadoqi, S. Kumar and Y. Yamada, J. Thermophys. Heat Transfer, 2002, 16, 193-199.
35 B. J. Garrison and R. Srinivasan, J. Appl. Phys., 1985, 57, 2909-2914.

36 J. H. Chun, K. T. Park, D. H. Jo, J. Y. Lee, S. G. Kim, S. H. Park, E. S. Lee, J.-Y. Jyoung and S. H. Kim, Int. J. Hydrogen Energy, 2011, 36, 8422-8428.

37 Y. Aoyama, K. Suzuki, Y. Tabe, T. Chikahisa and T. Tanuma, ECS Trans., 2015, 69, 743-752.

38 D. R. Baker, D. A. Caulk, K. C. Neyerlin and M. W. Murphy, J. Electrochem. Soc., 2009, 156, B991-B1003.

39 N. Nonoyama, S. Okazaki, A. Z. Weber, Y. Ikogi and T. Yoshida, J. Electrochem. Soc., 2011, 158, B416-B423.

40 U. Pasaogullari, C. Wang and K. S. Chen, J. Electrochem. Soc., 2005, 152, A1574-A1582.

41 J. H. Nam, K.-J. Lee, G.-S. Hwang, C.-J. Kim and M. Kaviany, Int. J. Heat Mass Transfer, 2009, 52, 2779-2791.

42 C. Simon, D. Kartouzian, D. Müller, F. Wilhelm and H. A. Gasteiger, J. Electrochem. Soc., 2017, 164, F1697-F1711. 